\title{
Cyclic Guanosine Monophosphate Measurement
}

National Cancer Institute

\section{Source}

National Cancer Institute. Cyclic Guanosine Monophosphate Measurement. NCI

Thesaurus. Code C111165.

The determination of the amount of cyclic guanosine 3,5-monophosphate present in a sample. 